\title{
ON SPECTRAL MODELS AND COLOUR CONSTANCY CLUES
}

\author{
Dmitry P. Nikolaev \\ Petr P. Nikolayev \\ Institute for Information Transmission Problems, RAS \\ Bol'shoj Karetnyj lane, 19, 127994, Moscow, Russia \\ E-mail: nikol@iitp.ru; dimonstr@iitp.ru
}

\section{KEYWORDS}

Colour constancy, spectral models, reflexes

\begin{abstract}
Properties of spectral models used in algorithms of colour constancy (CC) are studied. It is shown that the closure of such a model under multiplication is an important property allowing one to generate $\mathrm{CC}$ clues. New CC clues are built using the Gaussian model as an example. For multiplication-closed models, the role of reflexes is investigated.
\end{abstract}

\section{INTRODUCTION}

At present, the colour can be seen as an important feature for image interpretation. One of the areas of special interest in colour analysis is searching for particular objects in videos or indexing image databases. However, the perceived colour does not directly match the reflection spectra of the objects. It is important in the case when a target object has to be successfully detected regardless of the illumination and observation conditions, which aren't known a priori. This happens, in particular, when retrieving the same object captured under different illumination conditions or by different sensors.

The problems of invariant recognition of colour objects were, from the very beginning, related to so-called colour constancy (CC). The ability of the vision system of man and animals to estimate the reflective properties of surfaces in the case when the illumination chromaticity changes, that is, the $\mathrm{CC}$ phenomenon, as well as CC algorithms suitable for machine vision systems have been discussed quite explicitly (Nyuberg et al. 1971; Brill 1978; Forsyth 1990; D'Zmura and Iverson 1993; Finlayson et al. 2001; Finlayson and Schaefer 2001; Barnard et al. 2002).

Most CC algorithms suggested so far either explicitly or implicitly include the following stages:

1) Advancing an a priori assumption on the properties of the whole scene or separate objects.

2) Considering a certain logical consequence of this assumption, which allows estimating the parameters of scene illumination.

3) Calculating the illumination parameter estimates.

4) Estimating the colouration of the objects.
For the example of the "white patch» algorithm, the assumption is the presence of a white object in the scene and the logical consequence is a perfect match of the chromaticity of the brightest stimulus contained in the scene and the illumination chromaticity.

\section{SPECTRAL MODELS OF CC}

In the scope of CC problems, the term "spectral model" means such a subspace of the spectral space that a unique response corresponds to each of the possible model spectral stimuli. For this purpose, various models with a limited number of parameters are introduced for approximating the spectral characteristics of the vision process: the curves of source emission, and objects reflectance.

As demonstrated earlier (Nikolayev at al. 2006) the quality of colour estimation with the $\mathrm{CC}$ algorithms varies depending on the spectral model chosen. This is due to the fact that the fourth step of the algorithm actually solves the problem of estimating the ratio of two functions on the basis of their projections. Obviously, this problem becomes well posed only after the model is set. Furthermore, the introduction of the spectral model allows estimating the response of one sensor on the basis of that of another one, even in the case when their colour spaces are independent. Below we shall demonstrate the existence of CC clues (a priori assumptions on the properties of the scene), associated with the features of the colour model chosen.

Most researchers dealing with the CC problem use socalled linear spectral models (LSM) (Yilmaz 1962; Brill 1978; Maloney 1986). In a LSM, the space of spectral functions is confined to a $3 \mathrm{D}$ linear subspace of the function space. Interesting particular cases of LSM are models in which the bases are step-wise functions (socalled "banded spectral model" (Stiles and Wyszecki 1962; Land and McCann 1971; Nyuberg et al. 1971) or the functions of spectral sensitivity of the sensor (Lee et al. 1995). However, all these models have the same drawback: they cannot adequately describe stimuli of a high saturation (Maloney, 1986). This fact is quite obvious if taking into account that highly saturated stimuli of different hue are almost linearly independent. It is not the case for the Gaussian analytical spectral model we suggested earlier (Nikolayev 1985; Nikolaev and Nikolayev 2005; Nikolaev et al. 2006). However, the Gaussian model also has some specific 
shortcomings, which concern, for example, modelling purple hues. Let us now clarify what properties should a spectral model preferably possess and whether the "ideal" spectral model could be built.

\section{PROPERTIES OF SPECTRAL MODELS}

In the course of image formation, a spectral stimulus undergoes a number of transformations. Among these are: multiplication by a factor (propagation of nonfocused light), multiplication by a spectral curve (reflection off a surface), addition to a spectral curve (illumination mixing), projection to a spectral curve (sensor registration). The advantages of spectral models are, respectively, the closure under first three operations and the possibility of inverting the operator of projecting onto the $3 \mathrm{D}$ spectral basis. The latter implies the possibility of analytically restoring the spectral stimulus on the basis of the corresponding vector stimulus of a trichromatic sensor. This list of useful functionalities should be added with the possibility of modelling all the spectral curves accurate to the sensor metamerism and with the convenience of human interpretation of the model parameters.

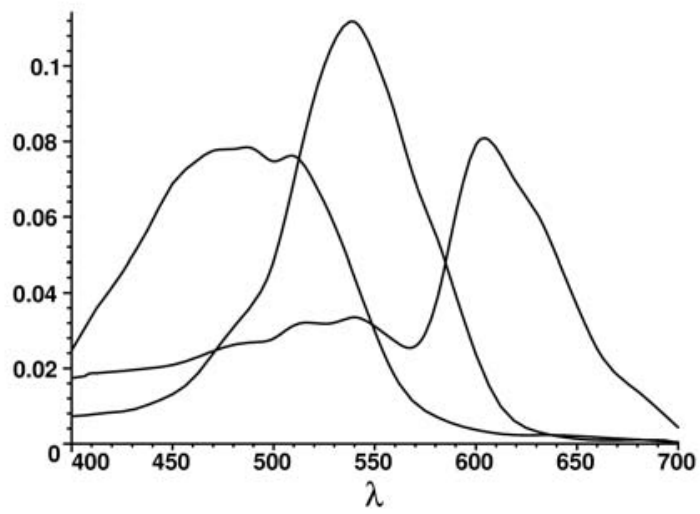

Figure 1: Spectral Sensitivity Curves of the Image Sensor Considered in Numeric Simulations

Obviously, not all of the above-mentioned features are equally important. The most important are the closure under multiplication by a factor (Property I) and spectral multiplication (Property II). Relevant experiments (Nikolaev et al. 2006) demonstrate that those models showing no closure under these two operations provide less accurate results. Apparently, this is due to additional errors arising in the course of secondary modelling of the spectra, after dividing the spectral stimulus by the source spectrum. The closure under addition (Property III) is not so important, because the stimulus sum can be most likely split into components in the linear space of vector stimuli, yet before transition to the spectral space. The next item of the list is colour-rendering completeness (Property IV), while analytical integrability (Property V) and intuitive clearness for human interpretation (Property VI) are evidently least important.
Consider now what spectral models can be obtained if requiring fulfilment of various subsets of the above properties. The spectral sensitivity curves of the sensor used for modelling are shown in Fig.1. Here, $\lambda$ is the radiation wavelength measured in nanometres. Figs. 2-5 show the colour-rendering completeness for different models. These figures use the chromaticity coordinates $(\alpha, \beta)$ defined as follows:

$\left\{\begin{array}{l}\alpha=(r-g) /(b+g+r) \\ \beta=(2 \cdot b-g-r) /(b+g+r) / \sqrt{3}\end{array}\right.$,

where $b, g$ and $r$ are the responses of the short-wave, medium-wave, and long-wave sensor channels, respectively.

\section{Classification of spectral models by their properties}

Obviously, fulfilment of Properties I and III signifies the linearity of the corresponding spectral model and causes Property $\mathrm{V}$ to be also fulfilled:

$F(\lambda, \vec{p})=p_{1} \cdot B_{1}(\lambda)+p_{2} \cdot B_{2}(\lambda)+p_{3} \cdot B_{3}(\lambda)$,

where $\vec{p}$ is the vector of model parameters and $B_{i}(\lambda)$ are the basis functions. To make Property II be true as well, it is necessary and sufficient to meet the following conditions:

$B_{i}(\lambda) \in\left\{0, c_{i}\right\}, \forall \vec{p}: \vec{p} \cdot \vec{B}(\lambda)=\operatorname{const}(\lambda)$

where $c_{i}$ are arbitrary constants. In fact, Eqs. (2)-(3) define a banded spectral model showing good results when modelling CC mechanisms (Nikolaev et al. 2006). The only considerable drawback of the banded spectral model is unsatisfactory colour rendering (see Fig.2), which is typical for all linear models.

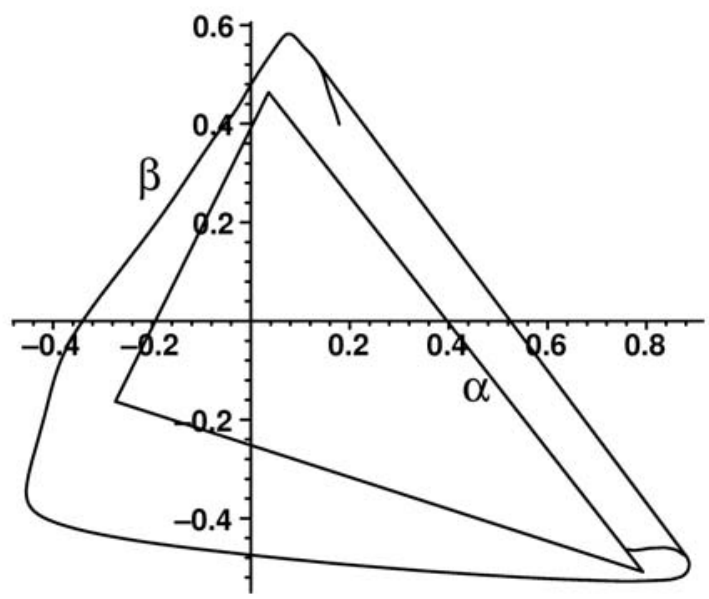

Figure 2: Banded Spectral Model Chart

Let us now try to reject Property III and to require meeting Properties II and IV. It follows from I and II that the logarithm of model spectra forms a linear subspace, such that one of the basis functions is constant. Choosing a second-order polynomial as the basis of this subspace, we obtain the Gaussian model:

$F(\lambda, \vec{p})=p_{1} \cdot \exp \left(-\frac{p_{2}}{2} \cdot\left(\lambda-p_{3}\right)^{2}\right)$. 


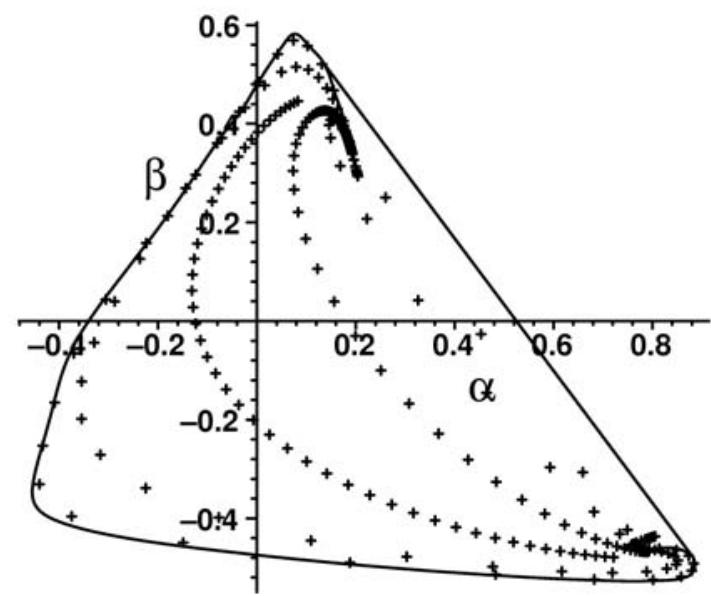

Figure 3: Gaussian Spectral Model Chart

As it can be seen from Fig.3, Property IV is well met for the Gaussian model. As mentioned earlier (Nikolaev et al. 2006), Properties V и VI are also true. However, this model approximates purple colours with spectra that exponentially increase at both boundaries of the visible wavelength range. This causes considerable instability of the model parameters in the course of modelling. Furthermore, the concept of a negative saturation implies no perfect intuitive clarity of the model parameters.

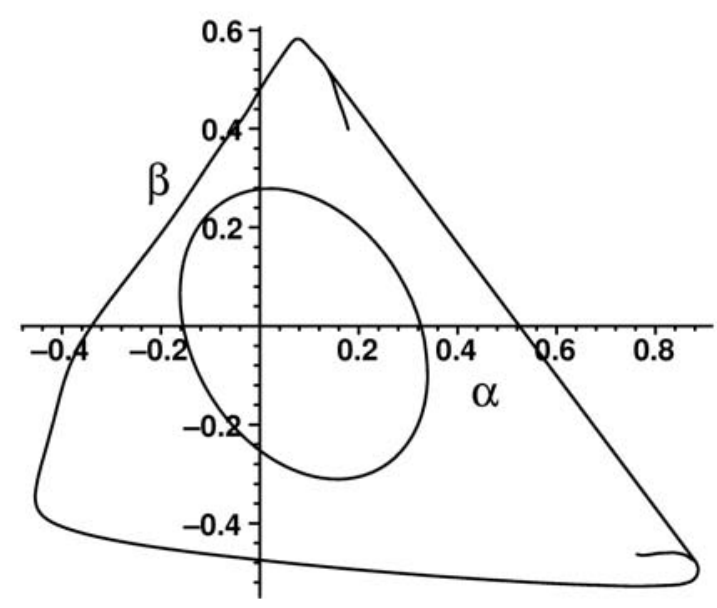

Figure 4: Yilmaz's Spectral Model Chart

Consider now one of the first spectral models ever proposed, Yilmaz's model:

$F(\lambda, \vec{p})=\frac{p_{1}}{1+p_{2}} \cdot\left(1+p_{2} \cdot \sin \left(\frac{2 \cdot \pi}{\Delta \lambda} \cdot\left(\lambda-p_{3}\right)\right)\right)$,

where $\Delta \lambda$ is the sensitivity range of the sensor. This model is linear $\left(B_{1}(\lambda)=1, B_{2}(\lambda)=\sin (2 \cdot \pi \cdot \lambda / \Delta \lambda)\right.$, $\left.B_{3}(\lambda)=\cos (2 \cdot \pi \cdot \lambda / \Delta \lambda)\right)$ and consequently meets Properties I, III, and V. Furthermore, its construction well satisfies Property VI: $p_{1}$ represents brightness, $p_{2}$ - saturation, and $p_{3}-$ hue. At the same time, the colour rendering provided by this model is far from perfection (see Fig. 4).

A similar construction performed for the signal logarithm provides a new model meeting Properties I, II, IV, and VI (let us refer to this model as Besselian):

$F(\lambda, \vec{p})=p_{1} \cdot \exp \left(-\frac{p_{2}}{2} \cdot\left(1+\sin \left(\frac{2 \cdot \pi}{\Delta \lambda} \cdot\left(\lambda-p_{3}\right)\right)\right)\right)$.

As it can be seen from Fig.5, Besselian model shows best results in colour rendering.

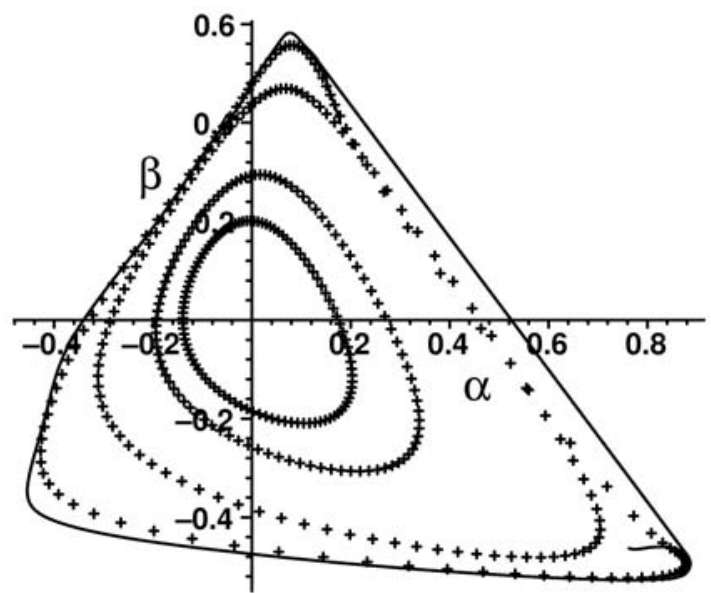

Figure 5: Besselian Spectral Model Chart

Table 1: Properties of Different Spectral Models

\begin{tabular}{|l|c|c|c|c|c|c|}
\hline Model & I & II & III & IV & V & VI \\
\hline General linear & + & $?$ & + & - & + & $?$ \\
\hline Banded (linear) & + & + & + & - & + & - \\
\hline Yilmaz's (linear) & + & - & + & - & + & + \\
\hline Gaussian & + & + & - & \pm & + & \pm \\
\hline Besselian & + & + & - & + & - & + \\
\hline
\end{tabular}

Table I summarizes the fulfilment of the abovementioned Properties for all the spectral models discussed. Symbols “+”, “ \pm " and "-_" mean fulfilment, partial fulfilment and omission respectively. Symbol "?" means dependence on model parameters.

\section{CLUES FOR MUTIPLICATION-CLOSED MODELS}

Consider now an interesting consequence of Properties I and II, which allows constructing CC clues for those spectral models satisfying these two properties. As already mentioned above, Properties I and II imply the linearity of the space of the logarithms of model spectra. Each spectrum satisfying the model corresponds to a vector, $\vec{p}^{\prime}$, in this space:

$\log \left(F\left(\lambda, \vec{p}^{\prime}\right)\right)=p_{1}^{\prime}+p_{2}^{\prime} \cdot B_{2}^{\prime}(\lambda)+p_{3}^{\prime} \cdot B_{3}^{\prime}(\lambda)$.

In particular, for the banded model $\widetilde{B}_{2}(\lambda)=B_{2}(\lambda)$, $\widetilde{B}_{3}(\lambda)=B_{3}(\lambda)$; for the Gaussian model $\widetilde{B}_{2}(\lambda)=\lambda$, 
$\widetilde{B}_{3}(\lambda)=\lambda^{2}$; for the Besselian model $\widetilde{B}_{2}(\lambda)=\sin (2 \cdot \pi \cdot \lambda / \Delta \lambda), \widetilde{B}_{3}(\lambda)=\cos (2 \cdot \pi \cdot \lambda / \Delta \lambda)$. Let us call such spectral models multiplication-closed.

For each pixel of an image, the vector $\vec{p}^{\prime}$ can be calculated on the basis of three known projections of the spectrum $F\left(\lambda, \vec{p}^{\prime}\right)$. This can be done either analytically (when Property V is met) or numerically (in any case). Since multiplication of spectra corresponds to addition in the logarithmic space, the distribution of the $\vec{p}^{\prime}$ vectors differs from that of the vectors corresponding to the reflective spectra of the scene objects by only a linear shift. In turn, the shift vector corresponds to the illumination spectrum.

For the models considered, any assumption on the kind of distribution of colours within the scene can serve as a $\mathrm{CC}$ clue. For instance, in the banded model the components of the $\vec{p}^{\prime}$ vector cannot exceed zero for reflective surfaces, while the coordinate origin corresponds to a white object. Assume that the scene contains some objects with $p_{i}^{\prime}=0$ («vividly coloured») ones). Then, $p_{i}^{\prime}$ will be maximal at the corresponding image pixels, which makes it possible to first find out these objects and then compute the illumination parameters. That's how the well-known "applique" algorithm is formulated.

Let us show now what a CC clue can be constructed for the Gaussian model. It is reasonable to suppose that the scene more often contains less saturated colours. For the Gaussian model, $\left|p_{3}\right|$ directly corresponds to the saturation, while $\left|p_{2}\right|$ corresponds to the saturation multiplied by the hue. Thus, we expect that the $\left(p_{2}, p_{3}\right)$ distribution reaches its maximum in the vicinity of the point $(0,0)$. As a result, we obtain the following CC algorithm. First, construct for the scene image its distribution $\left(p_{2}, p_{3}\right)$ in the Gaussian model. Then, calculate the medians, $\operatorname{med}\left[p_{2}\right]$ and $\operatorname{med}\left[p_{3}\right]$. The point $\left(\operatorname{med}\left[p_{2}\right], \operatorname{med}\left[p_{3}\right]\right)$ is then considered an estimate of the light source chromaticity.

Similarly, different CC clues can be constructed for the multiplication-closed models. However, the accuracy of their operation always depends on the adequacy of the statistical model of colour distribution across the scene. Let us now demonstrate how taking into account scene reflexes may reduce this dependence and enhance the stability of the clues.

\section{ROLE OF FOLDS FOR MUTIPLICATION- CLOSED MODELS}

The presence of folds on uniformly coloured surfaces causes reflexes to arise in the scene. The fold region becomes illuminated not only with the primary source but also with the opposite side of the fold. The stimulus produced by the fold can be expressed as:

$F(\lambda, \vec{r})=g_{1}(\vec{r}) \cdot S(\lambda) \cdot \Phi(\lambda)+g_{2}(\vec{r}) \cdot S(\lambda) \cdot \Phi^{2}(\lambda)+\ldots,(8)$

where $\vec{r}$ is the radius vector of a point of the fold and $g_{1}(\vec{r})>g_{2}(\vec{r}) \geq \ldots$ are coefficients depending on the scattering properties of the surface and the scene geometry. At the fold boundary $g_{2}(\vec{r})=g_{3}(\vec{r})=\ldots=0$. It can be assumed that no secondary illumination exists within the fold, while the colouration smoothly changes, according to the following expression:

$$
\Phi^{\prime}(\lambda, \vec{r})=\Phi(\lambda)+\frac{g_{2}(\vec{r})}{g_{1}(\vec{r})} \cdot \Phi^{2}(\lambda)+\frac{g_{3}(\vec{r})}{g_{1}(\vec{r})} \cdot \Phi^{3}(\lambda)+\ldots .
$$

Such a colouration remains in the framework of the spectral modal only in the case of the banded model. However, for some multiplication-closed models, Eq.(8) can be approximated as:

$$
\Phi^{\prime}(\lambda, \vec{r}) \approx g^{\prime}(\vec{r}) \cdot \Phi^{\alpha(\vec{r})}(\lambda),
$$

where $\alpha>1$. An example of such an approximation for the Gaussian model is shown in Fig.6. Here, one can see the $\Phi^{\prime}(\lambda)$ spectrum for $\Phi(\lambda)=\exp \left(-10^{-3} \cdot(\lambda-550)^{2}\right)$ and $g_{i}=4^{1-i}$, its approximation with the parameters $g^{\prime}=1.33, \alpha=1.2$, and the approximation error. As it can be seen from the figure, the approximation is accurate enough.

Thus, for those multiplication-complete models making approximation (10) adequate, the magnitudes of the parameters $\vec{p}_{2}^{\prime}$ and $\vec{p}_{3}^{\prime}$ proportionally grow within folds. This fact allows detecting folds on objects with nonzero saturation (Funt and Drew 1993).

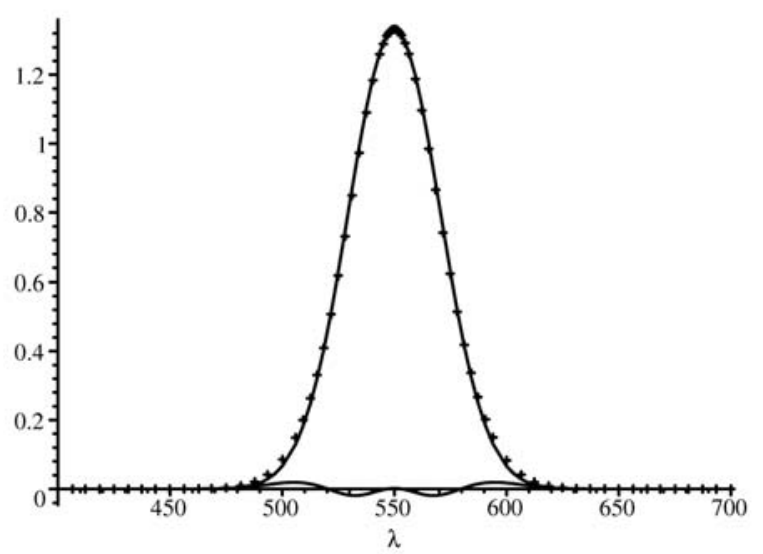

Figure 6: Approximation of a Gaussian Stimulus Within a Fold

An indication of a fold is a local increase in the rank of the object in the colour space (according to the terminology proposed in Ref. (Nikolaev and Nikolayev 2004)). At the same time, unlike the case of a highlight, no discontinuity of the stimulus intensity is observed. Let us now show how the properties of folds can be 
used. Consider an object, on whose surface a fold is presumably detected. Let the parameters of the object stimuli beyond the fold are $\left(p_{2}, p_{3}\right)$. The combination of the signs of the deviations $\left(\Delta p_{2}, \Delta p_{3}\right)$ of the stimulus parameters within the fold indicates the quadrant containing the colouration parameters, regardless of the illuminant-induced shift. So, each fold found superimposes two inequalities to the coordinates of an achromatic object, thereby narrowing the seek area and decreasing the maximum possible error.

Suppose now that the deviation $\left(\Delta p_{2}, \Delta p_{3}\right)$ is large enough to distinguish it against a noise background. Then, two correctly detected folds are enough to estimate the chromaticity of the scene illumination source. Indeed, distribution (8) within approximation (10) depicts on the $\left(p_{2}, p_{3}\right)$ plane a line segment crossing the point corresponding to $\alpha=0$, that is, the point portraying the illumination spectrum. Thus, two segments are enough to determine the illumination chromaticity. The corresponding plot is shown in Fig. 7. Solid gray circle corresponds to the chromaticity of the illuminant. Series of smaller circles correspond to colour distribution of the folds. Pigments in this numerical experiment were randomly chosen from database of natural pigments. One can see that colour distribution of the fold points on the $\left(p_{2}, p_{3}\right)$ to the position of the light source even in the case of nongaussian reflectances.

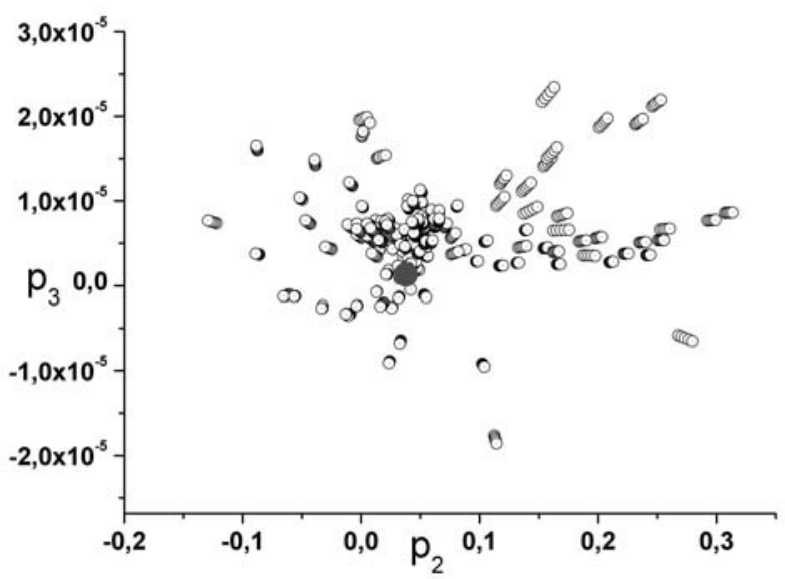

Figure 7: Illumination Estimation Using Two Folds

\section{CONCLUSION}

The results obtained make us once again return to the problem of adequacy of the spectral models used in colour machine vision. A properly chosen spectral model not only ensures a more accurate estimation of the colouration parameters of the scene objects but also allows constructing new features of the illumination chromaticity. The authors are going to continue studying the properties of multiplication-closed models, hoping for the discovery of new, more stable $\mathrm{CC}$ algorithms.

\section{ACKNOWLEDGEMENTS}

This work is partly supported by the Russian Foundation for Basic Research (grant № 06-06-80390a) and Grant of the President of the Russian Federation for Young Scientist Support (grant № MK4567.2006.9).

\section{REFERENCES}

Barnard, K.; L. Martin; A. Coath and B. Funt. 2002. "A comparison of colour constancy algorithms. Part two. Experiments with image data." IEEE Trans. in Image Processing 11, No. 9, 985-996.

Brill, M. 1978. "A device performing illuminant-invariant assessment of chromatic relations." J. Theor. Biology 71, No. 3, 473-478.

D’Zmura, M. and G. Iverson. 1993. "Colour constancy. I. Basic theory of two-stage linear recovery of spectral description for lights and surfaces." J. Opt. Soc. Amer. A. $10,2148-2165$.

Finlayson, G.D.; Hordley S. and Hubel P. 2001. "Colour by correlation: a simple, unifying framework for colour constancy". IEEE Trans. Pattern Anal. Mash. Intel. 23, No. 12, 1209-1221.

Finlayson, G.D. and G. Schaefer. 2001. "Solving for colour constancy using a constrained dichromatic reflection model". Int. J. Computer Vision 42, No. 3, 127-144.

Funt, B.V. and Drew M.S. 1993. "Color Space Analysis of Mutual Illumination". IEEE Trans. Pattern Anal. Mach. Intell. 15(12), 1319-1326

Forsyth, D.A. 1990. "A novel algorithm for colour constancy". Int. J. Computer Vision 5, No. 1, 5-36.

Land, E.H. and J.J. McCann. 1971. "Lightness and retinex theory". J. Opt. Soc. Amer., A 61, 1-11.

Lee, S.D; C.Y. Kim and Y.S. Seo. 1995. "Linear model of surface and scanner characterization method." In Proceedings of Device-Independent Colour Imaging II. SPIE, 2414, 84-93.

Maloney, E. 1986. "Evaluation of linear models of surface spectral reflectance with small number of parameters." $J$. Opt. Soc. Amer. 3, No. 10, 1673-1683.

Nikolayev, P.P. 1985. "Model of colour constancy for the case of continuous spectral functions". Biophysics 30, No 1, 112-117.

Nikolaev, D.P. and P.P. Nikolayev. 2004. "Linear color segmentation and its implementation." Computer Vision and Image Understanding 94, 115-139.

Nikolaev, D.P. and P.P. Nikolayev. 2005. "Comparative analysis of Gaussian and linear spectral models for colour constancy." In Proceedings of $19^{\text {th }}$ European Conference on Modelling and Simulation, 300-305.

Nikolaev, D.P.; P.P. Nikolayev and V.P. Bozhkova. 2006. "Efficiency comparison of analytical Gaussian and linear spectral models in the same colour constancy framework". International Journal of Simulation - Systems, Science \& Technology (IJSSST, Special Issue on: Vision \& Visualization) 7, No 3, 21-36.

Nyuberg, N.D.; P.P. Nikolayev and M.M. Bongard. 1971. "II. On the constancy of perception of colouration". Biophysics 16, No. 6, 1052-1063 (in Russian).

Stiles, W.S. and G.W. Wyszecki. 1962. "Counting metameric object colours". J. Opt. Soc. Amer 52, No. 2, 313-328.

Yilmaz, H. 1962. "The theory of colour vision". In Biological Prototypes and Synthetic Systems, E. Bernard and M. Kare (Eds.). N-Y, Plenum Press, 1, 126-143. 


\section{AUTHOR BIOGRAPHIES}

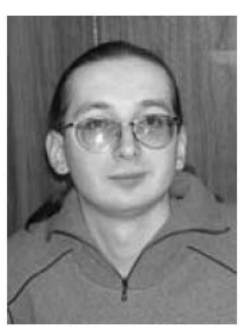

DMITRY P. NIKOLAEV was

born in Moscow, Russia. He studied physics, obtained his Master degree in 2000 and Ph.D. degree in 2004 from Moscow State University. Since 2000 he has been a research scientist at the Institute for Information Transmission Problems, RAS. His research activities are in the areas of computer vision with primary application to colour image understanding. His e-mail address is dimonstr@iitp.ru and his Web page can be found at http://www.ddt.ru/chaddt/Lace/pub.html.

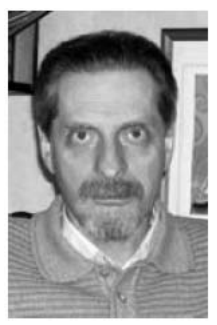

PETR P. NIKOLAYEV was born in Moscow, Russia. He studied physics and obtained his Master degree in 1966 from Moscow State University. He obtained a Ph.D. degree in biology in 1975 from the Institute for Information Transmission Problems, RAS and a Dr. of Science degree in physics in 1993 from the Institute for Biophysics, RAS. Since 1966, he has been a research scientist at the Institute for Information Transmission Problems, RAS, where he is now leading a research group in the field of computer colour vision. His e-mail address is nikol@iitp.ru. 